\title{
Gravity and Spin Dynamics for the EDM Search Experiments
}

\author{
N.N. Nikolaev* \\ L.D. Landau Institute for Theoretical Physics, 142432 Chernogolovka, Russia and Moscow \\ Institute of Physics and Technology, 141701 Dolgoprudny, Russia \\ E-mail: nikolaev@itp.ac.ru \\ F. Rathmann \\ Institut f. Kernphysik, FZJ, 52415 Juelich, Germany. \\ E-mail: F.Rathmannefz-juelich.de
}

\section{A. Saleev}

Institut f. Kernphysik, FZJ, 52415 Juelich, Germany.

E-mail: a.saleevafz-juelich.de

\section{A. Silenko}

N.N. Bogolyubov Laboratory for Theoretical Physics, JINR, 141980 Dubna, Russia and

Belarusian State University, 220030 Minsk, Belarus.

E-mail: alsilenko@mail.ru

\section{for the JEDI Collaboration}

\begin{abstract}
We review the General Relativity induced precession of a spin of relativistic particles in storage rings in terrestrial environment. Besides the clasical geodetic effect there emerges a spin rotation in focusing fields needed to compensate for the Earth gravity force acting on stored particles. The both effects have a structure of the spin-orbit interaction in the Earth gravitational field. Our major point is a vindication of the 2012 Orlov-Flanagan-Semertzidis derivation of the GR induced false EDM signal in the all-electric frozen-spin rings. The experimental observation of the GR signal would serve as an important indicator of a performance of a storage ring. We also comment on the GR effect in a hybrid $\vec{E} \times \vec{B}$ ring envisioned by the CPEDM Collaboration.
\end{abstract}

23rd International Spin Physics Symposium - SPIN2018 -

10-14 September, 2018

Ferrara, Italy

${ }^{*}$ Speaker. 


\section{Introduction}

Systematic background is a bane of high precision searches for the Electric Dipole Moment (EDM) of charged particles in storage ring experiments. The JEDI collaboration working at COSY ring in Juelich had recently obtained outstanding experimental results on spin dynamics of relevance to search for the EDM signal at all-magnetic storage rings. A comprehensive review of these results and their implications for the future all-electric storage rings was given in the plenary talk by F. Rathmann at this conference [1]. Here we only single out an important first ever in situ quantification of the false EDM signal from the rotation of the magnetic dipole moment (MDM) in the imperfection magnetic fields in the ring - while the imperfections proper were found to be quite substantial, the stable spin angular orientation at COSY can be determined to a $\mu \mathrm{rad}$ accuracy [2]. Such an evil magnetic imperfection fields will be absent in the all-electric storage rings. It is proper to advertise here that based on the JEDI achievements a new international CPEDM Collaboration has been formed aiming at a construction of the all-electric ring in Europe [3]. In this writeup we focus on still another magnetic imperfection induced in all-electric storage rings by the General Relativity (GR) effects. The presented material is part of a JEDI activity related to discussions of the CPEDM project. A correct treatment of the GR effects has been given by Orlov, Flanagan and Semertzidis in 2012 [4], but in 2017 it has been challenged by T. Morishima et al [5] what lead to quite an active dicussion of the GR effect (a fairly complete summary of this discussion was given by Lee Roberts [6], see also more recent [7]). The basic aim of this writeup is a clarification of some of the issues in spin dynamics in curved space-time of relevence to storage ring experiments and a vindication of the O-F-S result. A critical point is that the GR induced false EDM signal can readily be separated from the true EDM signal with counterrotating beams in an all-electric ring. Furthermore, the GR provides a unique reference signal for a crosscheck of the ring performance.

\section{FT-BMT equation}

The Schroedinger equation for spin dynamics in electromagnetic fields is with us since the classical works by Thomas [8] and Frenkel [9] on the celebrated 1/2 factor in the spin-orbit interaction in atoms. It has been put in the widely used modern form appropriate for storage rings by Bargmann, Michel and Telegdi [10]. With allowance for the EDM contribution to spin rotations the FT-BMT equation reads (here $\hbar=c=1$ ) $[11,12]$

$$
\begin{gathered}
\frac{d \vec{S}}{d t}=\left[\vec{\Omega}_{s} \times \vec{S}\right], \\
\vec{\Omega}_{s}=-\frac{q}{m}[\underbrace{G \vec{B}+\left(\frac{1}{\beta^{2}}-1-G\right) \vec{\beta} \times \vec{E}}_{M D M}+\underbrace{\frac{1}{2} \eta_{E D M}(\vec{E}+[\vec{\beta} \times \vec{B}])}_{E D M}] .
\end{gathered}
$$

It describes rotation of the spin w.r.t. the particle momentum and gives a dfference of the true spin precession and cyclotron precession angular velocities. Here $G=(g-2) / 2$ is the magnetic anomaly of a particle of charge $q$ and mass $m, \eta_{E D M}$ defines its EDM in units of the nuclear magneton

$$
d=\eta_{E D M} \frac{q}{2 m},
$$


and we consider a reference closed orbit with a pure vertical magnetic field $\vec{B}=B \vec{e}_{y}$, pure horizontal electric field $\vec{E}=E \vec{e}_{x}$ and $(\vec{\beta} \cdot \vec{E})=(\vec{\beta} \cdot \vec{B})=0$, where $\vec{\beta}=\beta \vec{e}_{z}$ is the particle velocity $\vec{v}$ in units of the velocity $c$ of light $\left(\left\{\vec{e}_{x}, \vec{e}_{y}, \vec{e}_{z}\right\}\right.$ form a right-handed coordinate system, $\vec{e}_{z}=\left[\vec{e}_{x} \times \vec{e}_{y}\right]$ ). Bear in mind that the above canonical form of the FT-BMT equation only holds for the pure electromagnetic confinement of particles in a ring.

The central idea is to eliminate the MDM effect altogether first by going to the all-electric ring, $\vec{B}=0$, and next operating at the frozen-spin (FS) magic energy [13]

$$
\beta^{2}=\frac{1}{1+G}
$$

Then the spin dynamics will boil down to a pure EDM driven rotation around the radial axis with the angular velocity

$$
\vec{\Omega}_{E D M}^{F S}=-\frac{\eta_{E D M} q}{2 m} \vec{E}
$$

and a slow build up of the vertical polarizaton from the initially longitidinal one is a sought for signal of EDM. The in-plane polarization of a particle can be frozen either along its momentum or at any fixed angle w.r.t. the momentum. In the latter case the longitidinal polarization would give rise to the EDM signal, while the readily measureable sideways component would serve as sort of a comagnetometer to maintain the frozen-spin condition.

Now we recall that our storage rings rest on the terrestial surface and stored particles are subject to gravitational field of the Earth. While in the neutron EDM experiments the gravity plays a major role in traps for ultracold neutrons, for a good reason no one has ever cared about a free fall of charged particles in storage rings. A point is that the beam automatically finds the equilibrium position such that the gravitational force is cancelled for by a Lorentz force from focusing fields. A gravity induced shift of the stable orbit is absolutely insignicant for all the practical purposes.

\section{Gyroscope in a gravitational field}

The first discussion of precession of gyroscopes in gravitational fields by de Sitter, often referred to as the geodetic effect, goes back to 1916 [14]. A gyroscope precession test of GR was suggested by Schiff in 1960 [15]. Schiff's predictions have been fully confirmed by the satellite Garvity Probe-B experiment ([16] and references therein). In the calssification of Khriplovich and Pomeransky, the geodetic effect is but a spin-orbit interaction in a gravitational field [17]. The Kh-P relativistic extension of the spin precession angluar velocity from the geodetic effect reads

$$
\vec{\Omega}_{L S}=\frac{2 \gamma+1}{\gamma+1}[\vec{\beta} \times \vec{g}]
$$

The vector free fall acceleration $\vec{g}$ should not be confused with the magnetic g-factor. Because $\vec{g}$ is proportional to a gradient of the gravitational field, this result for $\vec{\Omega}_{L S}$ is fully reminiscent of the one from LS interaction familiar from atomic physics. Indeed, $\vec{g} \propto \vec{r}$, where $\vec{r}$ is a radius-vector from the Earth center, and the cross product $[\vec{\beta} \times \vec{r}]$ gives rise to an orbital momentum $\vec{L}$ of a particle. The geodetic effect does not depend on the magnetic anomaly.

As far as the Earth gravity is concerned, is suffices to invoke the weak field approximation. 


\section{The S-T, O-S-T and O-F-S effects}

The Kh-P relativistic formula for a geodetic effect describes a spin precession in the free fall motion. In particle physics we deal with charged particles confined in a ring by static electromagnetic fields. Here we ask for a closed orbit in a ring placed on the terrestial surface and the gravity force

$$
\vec{F}_{g}=\frac{2 \gamma^{2}-1}{\gamma} m \vec{g}
$$

needs to be compensated by a Lorentz force from the focusing fields in the ring. The energy dependence of this gravity force is dictated by the spin-2 nature of gravitons. The rest of the story is somewhat different for the all-magnetic and all-electric fields. In the further discussion of the GR effects we prefer to follow a historic route.

Silenko and Teryaev were the first to discuss this compensation in the all-magnetic ring as early as in 2005 [18]. It is furnished by the radial magnetic field

$$
\vec{B}_{g}=\frac{2 \gamma^{2}-1}{\gamma v^{2}} \cdot \frac{m}{q}[\vec{v} \times \vec{g}] .
$$

Upon this compensation the cyclotron rotation will be exactly the same as in a pure vertical driving field, but the focusing field $\vec{B}_{r}$ shall emerge as an extra in the FT-BMT equation.

Although of apparent electromagnetic origin, a net effect of this GR correction to spin precession is of precisely the same form as the geodetic effect. The combined GR contribution to spin precession becomes

$$
\vec{\Omega}_{g M}^{S T}=-\frac{1}{\gamma v^{2}}\left\{1+G\left(2 \gamma^{2}-1\right)\right\}[\vec{v} \times \vec{g}]
$$

The S-T effect was a first instance of an interplay of the geodetic and focusing field effects, although from the point of view of the EDM the S-T formula (4.3) is of pure academic interest as in allmagnetic rings it is an absolutely negligible and basically unobservable effect.

The first treatmet of the GR effect on the EDM in all-electric frozen spin rings was given in 2012 by Orlov, Flanagan and Semertzidis [4]. As a heavy juggling by GR formalism it is not an easy reading and a separation of the geodetic and free-fall compensation effects might look elusive in there. The O-F-S final result reads (hereafter the velocity of light $\mathrm{c}$ is in)

$$
\vec{\Omega}_{g E}^{O F S}=\frac{|\vec{g}| \sqrt{G}}{c} \vec{e}_{r} .
$$

The case of all-electric storage ring was revisited in 2016 by Obukhov, Silenko and Teryaev [19]. In this case the free fall is compensated for by a vertical focusing electric field

$$
\vec{E}_{g}=-\frac{2 \gamma^{2}-1}{\gamma} \cdot \frac{m}{q} \vec{g} .
$$

In a generic ring running at a non-magic energy this vertical electric field amounts to a motional radial magnetic field $\propto[\vec{v} \times \vec{g}]$. One can say that the GR effects do always amount to generation of the radial magnetic imperfection fields. When combined with the geodetic effect, it gives the total GR induced angular velocity of spin rotation around the radial axis

$$
\vec{\Omega}_{g E}^{O S T}=\frac{1-G\left(2 \gamma^{2}-1\right)}{c^{2} \gamma}[\vec{v} \times \vec{g}] .
$$


At first sight, the O-S-T formula might look quite different from the O-F-S one, but once the frozenspin condition (2.4) is imposed in the O-S-T generic equation, the O-S-T result becomes identical to the O-F-S one.

Remarkably, the GR effect does not depend on the electric field in the ring.

\section{GR effects in the combined $\vec{E} \times \vec{B}$ ring for $G<0$}

For deuterons with $G<0$ the frozen spin condition can be met only in a hybrid ring with crossed electric and magnetic fields. The same is true of the prototype frozen-spin ring for low energy $45 \mathrm{MeV}$ protons. In a hybrid ring the GR correction to the spin precession would depend on the relative contribution of the magnetic and electric focusing fields to the Lorentz force

$$
\kappa=\frac{v B_{g}}{E_{g}} .
$$

This new parameter is not fixed by the frozen-spin condition of vanishing MDM rotation in confining fields. Then the GR induced false EDM signal would be

$$
\vec{\Omega}_{g}=\frac{1}{1+\kappa}\left(\vec{\Omega}_{g E}+\kappa \vec{\Omega}_{g M}\right)
$$

\section{When does the false GR signal take over the EDM signal?}

Go back to the all-electric frozen-spin ring storing protons with magic momentum of $\approx 0.701$ $\mathrm{GeV} / \mathrm{c}$. The electrostatic deflectors sustaining electric fields of $\sim 7 \mathrm{MV} / \mathrm{m}$ are considered feasible. Then the false signal from the GR induced MDM rotation would become equal to the EDM signal at $\eta \approx 4 \cdot 10^{-14}$, i.e., at $d_{p} \approx 4 \cdot 10^{-28} e \cdot \mathrm{cm}$. At still smaller $d_{p}$ the GR signal would take over.

While the true EDM signal is identical for the clockwise (CW) and counterclockwise (CCW) beams, a false GR signals are of opposite sign. Hence in a ring with $\mathrm{CW}$ and $\mathrm{CCW}$ beams the two effects can readily be separated. Furthermore, in experiments aiming at an ultimate sensitivity $d_{p} \sim 10^{-29} \mathrm{e} \cdot \mathrm{cm}$ the GR effect would become an indispensable calibration check.

We also reiterate that unlike the true EDM signal the false EDM-like contribution from the GR signal does not depend on the radial confining field. However, testing this property would require impracticaly expensive a comparison of results from two magic energy rings with different confining E-fileds and different radii.

\section{Conclusions}

On the theoretical side, the issue of GR induced false EDM signal has been closed. However, one should not view this false GR signal as a systematic error. Quite to the contary, if on the experimental side a senisitivity to the proton EDM will ever be boosted to the level of $d_{p} \sim 10^{-29} e$. $\mathrm{cm}$, then the gravity signal will be seen in full glory and, as it has become a folklore, can be taken for a standard candle ${ }^{1}$. Its observation would become a very important and uniqe confirmation of spin dynamics of relativistic particles in General Relativity.

\footnotetext{
${ }^{1}$ While a clearcut separation of the EDM and GR effects was commented on already by Orlov et al. [4], the term "standard candle" can, apparently, be attributed to R. Talman.
} 


\section{Acknowledgemens}

The authors wish to acknowledge a support of the JEDI collaboration by the European Research Council via the ERC Advanced Grant "srEDM" (No. 694340). The work of NNN is a part of the Russian State program 0033-2019-0005.

\section{References}

[1] F. Rathmann, Plenary talk at the 23rd International Spin Physics Symposium - SPIN2018, 10-14 September, 2018, to be published in PoS. Ferrara, Italy

[2] A. Saleev et al. [JEDI Collaboration], Phys. Rev. Accel. Beams 20, no. 7, 072801 (2017) [Phys. Rev. Accel. Beams. 20, 072801 (2017)]

[3] F. Abusaif et al., "Feasibility Study for an EDM Storage Ring," arXiv:1812.08535 [physics.acc-ph].

[4] Y. Orlov, Y. Flanagan and Y. Senetrzidis, Phys. Lett. A376, 2822 (2018)

[5] T. Morishima, T. Futamase and H. M. Shimizu, PTEP 2018, no. 8, 089201 (2018)

[6] J.P. Miller and B.L. Roberts, arXiv:1805.01944 [hep-ph].

[7] A. Laszlo and Z. Zimboras, Class. Quant. Grav. 35, no. 17, 175003 (2018) [arXiv:1803.01395 [gr-qc]].

[8] L.H. Thomas, Nature 117, 514 (1926)

[9] J. Frenkel. Z.Phys. 37, 243 (1926)

[10] V. Bargmann, L. Michel, V.L. Telegdi, Phys.Rev.Lett. 2, 435 (1959)

[11] D.F. Nelson, A.A. Schupp, R.W. Pidd, H.R. Crane, Phys.Rev.Lett. 2, 492 (1959)

[12] T. Fukuyama, A.J. Silenko, Int.J.Mod.Phys. A28, 1350147 (2013)

[13] V. Anastassopoulos et al., Rev.Sci.Instrum. 87, no.11, 115116 (2016)

[14] W. de Sitter, Mon. Not. R. Astron. Soc. 77, 155 (1916).

[15] L.I. Schiff, Phys. Rev. Lett. 4, 215 (1960)

[16] C.W.F. Everitt et al. Phys. Rev. Lett. 106, 221101 (2011)

[17] I. B. Khriplovich and A. A. Pomeransky, J. Exp. Theor. Phys. 86, 839 (1998) [Zh. Eksp. Teor. Fiz. 113, 1537 (1998)]; A. A. Pomeransky, R. A. Senkov and I. B. Khriplovich, Phys. Usp. 43, 1055 (2000) [Usp. Fiz. Nauk 43, 1129 (2000)].

[18] A. J. Silenko and O. V. Teryaev, Phys. Rev. D 71, 064016 (2005) [gr-qc/0407015].

[19] Y. N. Obukhov, A. J. Silenko and O. V. Teryaev, Phys. Rev. D 94, no. 4, 044019 (2016) 